\title{
Incidencia y factores de riesgo del recién nacido de muy alto peso en el Hospital Hipólito Unanue de Tacna
}

\section{Incidence and risk factors of very high birth weight newborn at Hipolito Unanue Hospital in Tacna}

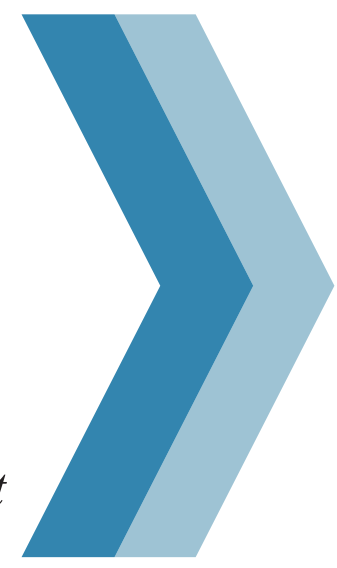

\author{
Beatriz Paola Luna Chuquiña ${ }^{1 a}$ \\ Manuel Ticona Rendón ${ }^{2 b}$ \\ Rubens Pérez Mamani ${ }^{3 \mathrm{c}}$ \\ ${ }^{1}$ Investigador independiente. Tacna, Perú. \\ ${ }^{2}$ Escuela de Medicina Humana de la Universidad Nacional Jorge Basadre. Tacna, Perú. \\ ${ }^{3}$ Escuela de Postgrado de la Universidad Nacional Jorge Basadre. Tacna, Perú. \\ ${ }^{a}$ Médico Cirujano. \\ ${ }^{\mathrm{b}}$ Docente, Doctor en Salud Pública. \\ ${ }^{c}$ Docente, Ingeniero Comercial
}

\section{RESUMEN}

El recién nacido de 4500 gramos a más presenta mayor morbimortalidad neonatal, tanto como en su vida futura. Objetivo: Determinar incidencia y factores de riesgo del recién nacido de muy alto peso al nacer en el Hospital Hipólito Unanue de Tacna. Material y Método: Estudio analítico de casos y controles. Se incluyó a todos los recién nacidos del Hospital Hipólito Unanue de Tacna desde 2008 al 2017 que ascienden a 35236 . Los casos fueron recién nacidos vivos con peso $\geq 4500$ gramos siendo 859 en total y los controles recién nacidos vivos con peso entre 3000-3999 gramos equivalente a 24 638. Se utilizó estadísticos Odds Ratio (OR) con intervalo de confianza, y nivel de significación estadística con $\mathrm{p}<$ 0,05 . La información se obtuvo de la base de datos del Sistema Informático Perinatal (SIP). Para el procesamiento y análisis estadístico, se utilizaron los paquetes estadísticos Epi lnfo y SIP2000. Resultados: De 35236 partos, 859 presentaron recién nacidos de muy alto peso al nacer con una incidencia de $2,4 \%$. Los factores de riesgos biológicos fueron: antecedente de recién nacido de alto peso $\geq 4000 \mathrm{~g}(\mathrm{OR}=12,3)$, obesidad $(\mathrm{OR}=5,2)$, ganancia de peso materno $>16 \mathrm{~kg}(\mathrm{OR}=3,3)$, sobrepeso $(\mathrm{OR}=2,2)$ y edad materna de 35 años a más $(\mathrm{OR}=1,9)$. Los factores obstétricos fueron: diabetes materna $(\mathrm{OR}=5,4)$, polihidramnios $(\mathrm{OR}=$ $5,3)$, enfermedad hipertensiva del embarazo (OR = $1,9)$ y gran multípara $\geq 5$ partos $(\mathrm{OR}=1,7)$. Los factores perinatales asociados fueron: examen físico por Capurro $\geq 42$ semanas $(O R=8,2)$ y sexo de recién nacido masculino $(\mathrm{OR}=1,8)$. Conclusiones: $\mathrm{La}$ incidencia de recién nacidos de muy alto peso al nacer fue elevada y los factores de riesgo fueron principalmente maternos.

Palabras clave: Muy alto peso al nacer, macrosomía al nacer, obesidad infantil.

\section{ABSTRACT}

The newborn of 4500 grams or more has greater neonatal morbidity and mortality, as well as in his future life. Objective: To determine the incidence and risk factors of very high birth weight newborns at Hipólito Unanue Hospital in Tacna. Material and Method: Analytical study of cases and controls. All newborns of the Hipólito Unanue de Tacna Hospital from 2008 to 2017 amounting to 35,236 were included. The cases were live newborns weighing $\geq 4,500$ grams being 859 in total and the live newborn controls weighing between 3000- 3999 grams equivalent to 24638 . It was used Odds Ratio (OR) statistics with confidence interval, and level of statistical significance with $\mathrm{p}<0.05$. The information was obtained from the Perinatal Computing System (SIP) database. For statistical processing and analysis, it was used the Epi lnfo and SIP2000 statistical packages. Results: Of 35236 deliveries, 859 had very high birth weight newborns with an incidence of $2.4 \%$. The biological risk factors were: history of high-weight newborn $\geq 4000 \mathrm{~g}$ (OR = $12.3)$, obesity $(\mathrm{OR}=5.2)$, maternal weight gain $>16$ $\mathrm{kg}(\mathrm{OR}=3.3)$, overweight $(\mathrm{OR}=2.2)$ and maternal age from 35 years to more $(\mathrm{OR}=1.9)$. Obstetric factors were: maternal diabetes $(\mathrm{OR}=5.4)$, polyhydramnios $(\mathrm{OR}=5.3)$, hypertensive disease of pregnancy $(\mathrm{OR}=1.9)$ and grand multiparous $\geq 5$ births $(\mathrm{OR}=1.7)$. The associated perinatal factors were: physical examination by Capurro $\geq 42$ weeks $(\mathrm{OR}=8.2)$ and male newborn sex $(\mathrm{OR}=1.8)$. Conclusions: The incidence of very high birth weight newborns was high and the risk factors were mainly maternal.

Keywords: Very high birth weight, macrosomia at birth, childhood obesity. 


\section{Introducción}

Los recién nacidos con crecimiento intrauterino excesivo representan un grupo heterogéneo y por ello de vital relevancia. El peso es una variable importante para la evaluación del estado de salud del neonato, ya que constituye un factor en la supervivencia, el crecimiento y el desarrollo. La macrosomía o macrosomatia, etimológicamente significa tamaño grande del cuerpo (1).

El Colegio Americano de Ginecología y Obstetricia define a la macrosomía como el peso igual o superior a $4500 \mathrm{~g}$ al nascimiento; otros autores emplean el percentil 90 del peso fetal para la edad gestacional para considerar macrosomía y también al producto con peso neonatal mayor a $4 \mathrm{~kg}$, el cual se asocia a un mayor riesgo relativo de morbilidad materna y neonatal. La macrosomía se asocia a una mayor morbilidad, mortalidad infantil y materna, influyendo notablemente la cesárea con una relación 2:1 de los partos eutócicos (2).

Muchos elementos influyen en el crecimiento y desarrollo intrauterino, además de actuar como determinantes tanto del desarrollo como del funcionamiento placentario. Estos factores son de tipo fetal como la gemelaridad, anomalías cromosómicas, malformaciones congénitas; factores de tipo ambientales y maternos como las enfermedades asociadas a la gestación, paridad, edad materna, estado nutricional entre otros (3).

La macrosomía adquiere relevancia al estar asociada a enfermedades maternas como diabetes tipo II e hipertensión arterial, edad materna superior a los 35 años, obesidad previa al embarazo, así como ganancia excesiva de peso durante el embarazo, antecedente de multiparidad y la postmadurez (2).

El primer reporte de macrosomía fetal en la literatura fue hecho por el monje médico Francois Rabelais en el siglo XVI, quien relató la historia del bebé gigante Gargantúa. Muchos años después, la esposa de Gargantúa murió al parir a Pantagruel "porque era tan asombrosamente grande y pesado que no podía venir al mundo sin sofocar a su madre".

Macrosomía o Macrosomatia (macro:'grande'; soma:'cuerpo'), etimológicamente significa tamaño grande del cuerpo. Tradicionalmente, la macrosomía fetal ha sido definida por un peso arbitrario al nacer, de aproximadamente 4000 ó 4500 gramos. El parto de estos fetos grandes ocasiona traumatismo tanto en la madre como en el feto.

Históricamente, la macrosomía fetal ha estado asociada a una alta tasa de morbilidad y mortalidad materna y perinatal, dos veces mayor que la morbimortalidad de la población general (4).

El objetivo del presente estudio fue conocer la incidencia y factores de riesgos de los recién nacidos de muy alto peso al nacer en el Hospital Hipólito Unanue de Tacna durante los años 2008 a 2017.

\section{Material y métodos}

Estudio epidemiológico y analítico de casos y controles. Se incluyó a todos los recién nacidos del Hospital Hipólito Unanue de Tacna desde 2008 al 2017, los cuales ascienden a 35 236. Los casos fueron todos los recién nacidos vivos con peso $\geq 4500$ gramos; es decir, 859. Los controles estuvieron conformados por todos los recién nacidos vivos con pesos entre $3000-3$ 999 gramos equivalente a 24638 .

Se utilizaron los estadísticos Odds Ratio (OR) con intervalo de confianza para muestras independientes, y se determinó el nivel de significación estadística con $\mathrm{p}<0,05$ (significativo) y de $\mathrm{p}<0,01$ (altamente significativo o muy significativo). Los datos se obtuvieron de la base de datos del Sistema Informático Perinatal (SIP). Para el procesamiento y análisis estadístico, se utilizaron los paquetes estadísticos Epi lnfo 6 y SIP2000.

\section{Resultados}

En el Hospital Hipólito Unanue de Tacna durante el periodo 2008 a 2017, acudieron 859 gestantes con recién nacidos de muy alto peso al nacer que recibieron atención de parto.

En la Tabla y Figura 1 se observa la incidencia total $(2,4 \%)$ de recién nacidos $(\mathrm{RN})$ de muy alto peso al nacer en el Hospital Hipólito Unanue de Tacna durante el periodo de 10 años (2008-2017). En referida tabla se puede observar que la mayor incidencia de recién nacidos de muy alto peso se dio en el año 2012 con $3,1 \%$ y la mínima incidencia se aprecia en el año 2008 con 1,8 $\%$. En general, en el periodo de los 10 años, se aprecia una tendencia sin variación. 
Tabla 1. Incidencia de recién nacidos de muy alto peso al nacer en el Hospital Hipólito Unanue de Tacna del 2008-2017

\begin{tabular}{|c|c|c|c|}
\hline Años & n de recién nacidos & $\begin{array}{l}\mathrm{n} \text { de recién nacidos de } \\
\text { muy alto peso al nacer }\end{array}$ & $\begin{array}{c}\text { Incidencia } \\
\%\end{array}$ \\
\hline 2008 & 3571 & 64 & 1,8 \\
\hline 2009 & 3701 & 88 & 2,4 \\
\hline 2010 & 3549 & 82 & 2,3 \\
\hline 2011 & 3408 & 92 & 2,7 \\
\hline 2012 & 3559 & 111 & 3,1 \\
\hline 2013 & 3617 & 90 & 2,5 \\
\hline 2014 & 3386 & 95 & 2,8 \\
\hline 2015 & 3565 & 99 & 2,8 \\
\hline 2016 & 3390 & 70 & 2,1 \\
\hline 2017 & 3490 & 68 & 1,9 \\
\hline Total & 35236 & 859 & 2,4 \\
\hline
\end{tabular}

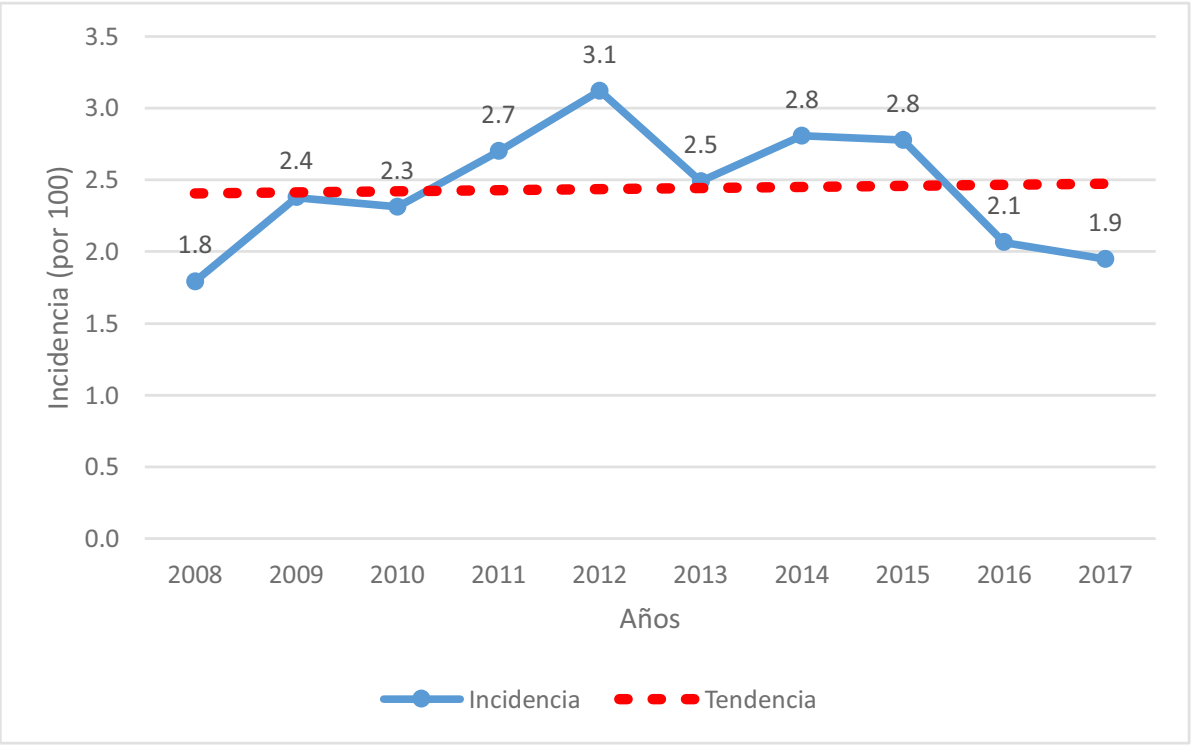

Figura 1. Incidencia y tendencia de muy alto peso al nacer en el Hospital Hipólito Unanue de Tacna, 2008-2017

En la Tabla 2, se observa que las madres de 35 años a más presentaron 1,9 veces mayor riesgo de tener recién nacidos de muy alto peso en comparación con las madres de 20 a 34 años de edad, siendo este riesgo estadísticamente significativo $(\mathrm{p}<0,01)$. En cuanto al estado nutricional pregestacional, se encontró que las madres con IMC de 25-29,9 tienen 2,2 veces mayor riesgo de tener recién nacidos de muy alto peso que las madres que tuvieron IMC de 18,5-24,9. Además, aquellas que tuvieron IMC de 30 a más presentaron un riesgo de 5,2, siendo ambos riesgos estadísticamente significativos ya que el $p<0,01$. En cuanto a la ganancia de peso, las que presentaron ganancia de peso mayor a
$16 \mathrm{~kg}$ tuvieron 3,3 veces mayor riesgo de tener recién nacidos de muy alto peso que las madres de 8 a $16 \mathrm{~kg}$ con asociación significativa $(\mathrm{p}<0,01)$. En cuanto al antecedente de recién nacido de alto peso, los recién nacidos que presentaron tales antecedente tuvieron 12,3 veces mayor riesgo de tener recién nacidos de muy alto peso que las madres que no tuvieron dicho antecedente con asociación significativa $(\mathrm{p}<0,01)$. En cuanto a la paridad, se encontró que las mujeres que tuvieron de 5 a más partos, presentaron 1,7 veces mayor riesgo de tener recién nacidos de muy alto peso que las madres que tuvieron 2-4 partos con asociación significativa $(\mathrm{p}<$ $0,01)$. 
Tabla 2. Factores de riesgo obstetricos asociados a muy alto peso al nacer en el Hospital Hipólito Unanue de Tacna, 2008-2017

\begin{tabular}{lccccccc} 
& Muy alto & Peso & & & & \\
\multicolumn{1}{c}{ Factores obstetricos } & peso & adecuado & OR & & IC $\%$ & P \\
& $\%$ & $\%$ & & Inf & Sup & \\
Edad materna $\geq 35$ & 23,9 & 12,7 & 1,9 & 1,6 & 2,3 & $<0,01$ \\
Gran multípara $\geq 5$ partos & 6,5 & 2,9 & 1,7 & 1,3 & 2,3 & $<0,01$ \\
Antecedente recién nacido $\geq 4000 \mathrm{~g}$ & 42,5 & 5,7 & 12,3 & 10,6 & 14,2 & $<0,01$ \\
Obesidad pregestacional imc $\geq 30$ & 39,0 & 15,8 & 5,2 & 4,3 & 6,2 & $<0,01$ \\
Sobrepeso pregestac. Imc $25-29,9$ & 37,8 & 35,8 & 2,2 & 1,8 & 2,6 & $<0,01$ \\
Ganancia de peso materno $>16 \mathrm{~kg}$ & 42,1 & 18,0 & 3,3 & 2,8 & 3,8 & $<0,01$
\end{tabular}

Las principales patologías maternas, en el periodo 2008-2017, fueron anemia 27,8 \%, infección urinaria $24,7 \%$ y enfermedad hipertensiva del embarazo $3,1 \%$. Siendo que la diabetes, polihidramnios y la enfermedad hipertensiva tuvieron 5,$4 ; 5,3$ y 1,9 veces más riesgo respectivamente de presentarse en un recién nacido de muy alto peso; las demás complicaciones no tuvieron significancia.

Tabla 3. Frecuencia y riesgo de recién nacidos de muy alto peso según patologías maternas

\begin{tabular}{lccccccc}
\multicolumn{7}{c}{ Muy alto peso } & \multicolumn{2}{c}{ Peso adecuado } & & \\
Patologías maternas & $\mathrm{n}$ & $\%$ & $\mathrm{n}$ & $\%$ & OR & IC & $\mathrm{P}$ \\
& 4 & 0,5 & 22 & 0,1 & 5,4 & $1,9-15,8$ & $<0,01$ \\
Diabetes & 3 & 0,4 & 17 & 0,1 & 5,3 & $1,5-18,0$ & $<0,01$ \\
Polihidramnios & 27 & 3,1 & 434 & 1,8 & 1,9 & $1,3-2,8$ & $<0,01$ \\
Enf. Hipertens. Emb & & & & & & & \\
Hiperemesis & 5 & 0,6 & 134 & 0,5 & 1,1 & $0,5-2,7$ & 0,82 \\
gravídica & 239 & 27,8 & 7031 & 28,5 & 1,01 & $0,8-1,2$ & 0,88 \\
Anemia & 212 & 24,7 & 6631 & 26,9 & 0,9 & $0,8-1,1$ & 0,30 \\
Infección urinaria & 2 & 0,2 & 185 & 0,8 & 0,3 & $0,1-1,3$ & 0,08 \\
Oligoamnios & & & & & & &
\end{tabular}

Tabla 4. Factores de riesgo neonatales asociados a recién nacidos (rn) de muy alto peso al nacer en el Hospital Hipólito Unanue de Tacna, 2008-2017

\begin{tabular}{|lccccccc} 
& $\begin{array}{c}\text { Muy alto } \\
\text { peso }\end{array}$ & $\begin{array}{c}\text { Peso } \\
\text { adecuado }\end{array}$ & OR & \multicolumn{2}{c|}{ IC 95 \% } & \\
\hline & $\%$ & $\%$ & & Inf & Sup & P \\
\hline Edad gestacional $>41$ semanas & 0,9 & 0,1 & 8,2 & 3,7 & 18,1 & $<0,01$ \\
Sexo rn masculino & 64,4 & 50,2 & 1,8 & 1,6 & 2,1 & $<0,01$
\end{tabular}




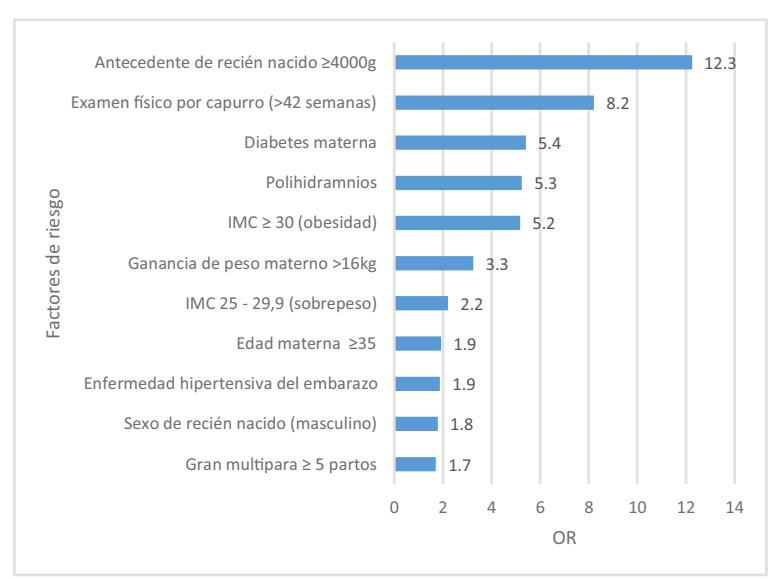

Figura 2. Factores de riesgo asociados a muy alto peso al nacer en el Hospital Hipólito Unanue de Tacna, 2008-2017

\section{Discusión}

El impacto de los factores de riesgo del recién nacido macrosómico $\geq 4000 \mathrm{~g}$ han sido estudiados ampliamente. Sin embargo, este es el primer estudio que se realiza en Perú sobre los factores de riesgo de recién nacidos de muy alto peso $(\geq 4500 \mathrm{~g})$.

La incidencia del recién nacido de muy alto peso al nacer en el Hospital Hipólito Unanue de Tacna durante el periodo de 10 años (2008-2017) tiene una tasa acumulada de $2,4 \%$ de recién nacidos vivos.

A nivel internacional tenemos el estudio de Heiskanen (5) realizado en el Hospital Universitario de Kuopio en Finlandia entre 1989 y 2001. Los autores encontraron 26961 embarazos únicos, de los cuales 886 madres dieron a luz a bebés $\geq 4500 \mathrm{~g}$, se compararon con los de 26075 bebes recién nacidos $<4500 \mathrm{~g}$. La incidencia de macrosomía fetal $\geq 4500 \mathrm{~g}$ o muy alto peso al nacer fue de 3,4\%, tal incidencia resulta superior a la presentada en nuestro estudio.

Por otro lado, Stotland et al. (6) realizaron un estudio en los hospitales del Programa de Cuidado Médico Permanente Kaiser en la Región del Norte de California, donde identificaron 146526 nacidos vivos entre 1995 y 1999 en una base de datos. Los investigadores analizaron factores de riesgo y las complicaciones asociadas con la macrosomía (peso $\geq$ $4500 \mathrm{~g}$ ) y encontraron que la tasa de macrosomía en la cohorte fue de $2,4 \%$ además la tasa de macrosomía se mantuvo estable durante el período de estudio de 5 años. Este estudio presentó una incidencia igual a la de nuestro estudio, con una tendencia lineal. Sin embargo la duración de este estudio ( 5 años) es inferior al nuestro (9 años).

Nassar et al. (7) realizaron un estudio en la Universidad Americana de Beirut Medical Center en Líbano, entre los años 1984 a 1996. Los investigadores encontraron a 231 madres con recién nacidos $\geq 4500 \mathrm{~g}$, cuya prevalencia fue de $1,5 \%$ durante los 13 años de estudio.

En la tabla 2, la edad materna en nuestro estudio se encontró que en el periodo 2008-2017 la razón entre recién nacidos de muy alto peso y los de normopeso al nacer es de 1,9 veces mayor en el grupo de puérperas añosas $\geq 35$ años, siendo significativa la asociación ( $\mathrm{p}<$ 0,01 ). Esto ya ha sido demostrado por varios autores que notaron que la edad avanzada era un factor de riesgo.

Heiskanen (5) en Finlandia encontró que las madres $\geq 35$ años fueron significativamente más propensas a tener macrosómicos $(\geq 4500 \mathrm{~g})$ que los recién nacidos con normopeso y menos probabilidad si es $<18$ años, lo cual es similar a nuestro estudio. Nkwabong et al. (8), en el Hospital Docente de la Universidad de Yaoundé y Maternidad central en Camerún desde el 1 de octubre del 2012 hasta el 30 junio de 2013, encontraron que la edad $\geq 30$ años tienen 3,8 veces más riesgo de tener recién nacido de muy alto peso que aquellas $<30$ años. En California, Stotland et al. (6) encontró que la edad materna de 30 a 39 años tiene 1,16 veces más riesgo de tener un recién nacido $\geq$ 4500 g que una madre de 20 a 29 años, siendo significativa la asociación $(\mathrm{p}<0,001)$.

En cuanto al estado nutricional pregestacional evaluado según índice de masa corporal, se encontró que aquellas gestantes con un IMC de 25 a 29,9 (sobrepeso) tienen 2,2 veces más de riesgo de presentar un recién nacido de muy alto peso al nacer en comparación con las gestantes de IMC 18,5 a 24,9; mientras las que tuvieron un IMC $\geq 30$ (obesidad) tienen 5,1 veces más riesgo de tener un recién nacido de muy alto peso al nacer, ambas con asociación significativa ( $\mathrm{p}$ $<0,001)$.

En Camerún, Nkwabong et al. (8) encontraron que los bebes macrosómicos fueron más frecuentes entre las mujeres con IMC promedio de $27 \mathrm{~kg} / \mathrm{m} 2$. En otro estudio, Heiskanen et al. (5) encontraron que un IMC pregestacional $\geq 25 \mathrm{~kg} / \mathrm{m} 2$ se presenta como factor de riesgo y las gestantes que presentaron IMC $\geq 30$ tuvieron 1,5 veces más de riesgo de presentar recién nacidos de muy alto peso al nacer que las que tenían $<25$ $\mathrm{kg} / \mathrm{m} 2$.

Esto demuestra que las mujeres obesas tienen un mayor riesgo de dar a luz a bebes macrosómicos $(\geq$ $4500 \mathrm{~g}$ ) que aquella con IMC normal.

Referente a la ganancia de peso, en nuestro estudio se encontró que aquellas puérperas que ganaron $>16 \mathrm{~kg}$ durante el embarazo tenían 3,2 veces más de riesgo de tener un recién nacido de muy alto peso $(\geq$ 
$4500 \mathrm{~g})$. Esto ya lo han encontrado algunos autores que observaron que el aumento excesivo de peso durante el embarazo era un factor de riesgo.

Nkwabong et al. (8), encontró que el aumento de peso de $\geq 16 \mathrm{~kg}$ tiene 4,2 veces mayor riesgo que las que tuvieron $<16 \mathrm{~kg}$, Por lo que se encontró que el aumento de peso materno durante el embarazo es un factor de riesgo para bebes macrosómicos ( $\geq 4500 \mathrm{~g})$. Esto significa que un mayor aporte nutricional durante el embarazo también podría ser un factor de riesgo para la macrosomía.

Respecto al antecedente de recién nacido de alto peso ( $\geq 4000 \mathrm{~g})$, encontramos que existe 12,3 veces más riesgo de tener bebes macrosómicos en aquellas que tienen antecedente de recién nacido de alto peso en comparación que las que no tenían el antecedente, siendo significativa la asociación $(\mathrm{p}<0,01)$. Esto ya lo han encontrado algunos autores como es el caso de Jiménez (9), en su estudio encontró que aquellos recién nacidos entre 4500 g-4 999 g, el $35 \%$ tenían antecedente de macrosomía.

Nkwabong et al. (8), encontró que las mujeres con antecedente de recién nacido $\geq 4000 \mathrm{~g}$ tienen 1,9 veces más riesgo de tener macrosómicos. $(\mathrm{OR}=1,9, \mathrm{IC}$ $95 \% 0,9-4,1)$. Esto no es sorprendente dado que en la misma mujer el peso al nacer generalmente aumenta en los embarazos siguientes. Una mujer que ha dado a luz a un bebé de $4100 \mathrm{~g}$ puede dar a luz otro de $\geq 4500 \mathrm{~g}$ en los embarazos posteriores.

Con respecto a la paridad, en nuestro estudio se encontró que las gran multíparas (partos $\geq 5$ ) tendrían 1,7 veces más riesgo de presentar un recién nacido de muy alto peso comparado con aquellas que tuvieron $2 \mathrm{a}$ 4 partos $(\mathrm{OR}=1,71, \mathrm{IC} 95 \% 1,28-2,27)$.

Jiménez et al. (9) encontraron que entre aquellos recién nacidos entre $4500 \mathrm{~g}-4999 \mathrm{~g}$, el 49,6\% eran descendientes de puérperas multíparas. Nassar (7) en Líbano, entre los años 1984 a 1996, encontraron 231 madres con recién nacidos $\geq 4500 \mathrm{~g}$ de los cuales el 81,4 $\%$ de los casos eran multíparas. Stotland et al. (6) encontraron que la multiparidad es factor de riesgo y predictor de macrosomía ( $\geq 4000 \mathrm{~g} \mathrm{y} \geq 4500 \mathrm{~g}$ ) con OR $=1,65$ y $\mathrm{OR}=1,75$ respectivamente, ambos con asociación significativa.

Sobre la morbilidad materna, no se encontró que sea factor de riesgo la presencia de morbilidad. Sin embargo, la presencia de diabetes es un factor de riesgo de recién nacido de muy alto peso al nacer $(\mathrm{OR}=5,411$ IC $95 \%$ 1,86 - 15,78). Esto es concordante con varios autores.

Jiménez et al. (9) encontraron que aquellos recién nacidos con peso entre $4500 \mathrm{~g}-4999 \mathrm{~g}$, el 42,3\% tiene diabetes. Oster y Toth (10) realizaron un estudio, cuyos datos fueron adquiridos del Programa de Salud Perinatal Alberta (APHP). En ese contexto, los autores encontraron que la diabetes materna es factor de riesgo de recién nacidos de muy alto peso al nacer $(\mathrm{OR}=2,181$ IC 95\% 1,77 - 2,68). Stotland et al. (6) encontraron que la diabetes es factor de riesgo y predictor de macrosomía $(\geq 4000 \mathrm{~g} \mathrm{y} \geq 4500 \mathrm{~g}) \operatorname{con}(\mathrm{OR}=1,7$ y $\mathrm{OR}=$ 2,50 respectivamente). Heiskanen et al. (5) encontraron que la diabetes materna presentó asociación fuerte con macrosomía (OR 4,6, IC 95 \% 2,57-8,24).

En cuanto a la enfermedad hipertensiva del embarazo en nuestro estudio se encontró que es factor de riesgo para recién nacido de muy alto peso al nacer (OR 1,9 IC $95 \%$ 1,26-2,81). Lo que difiere con otros autores como Oster y Toth (10); al igual que la hipertensión preexistente, los autores no encontraron que sea factor de riesgo. En cuanto a polihidramnios en nuestro estudio se encontró que es factor de riesgo para recién nacido de muy alto peso al nacer (OR 5,25 IC 95 $\% 1,53-17,97)$.

Sohaey et al. (11) realizo un estudio en Hospital Medical Center Swedish, sobre polihidramnios idiopáticos durante un período de 3 años (desde enero de 1989 hasta diciembre de 1991). En referido estudio, se evaluaron 113 fetos normales consecutivos con diagnóstico de polihidramnios idiopáticos mediante ecografía prenatal. El polihidramnios idiopático se estudió con ultrasonido (US) en 99 fetos normales consecutivos de mujeres no diabéticas. Posteriormente, se compararon los pesos al nacer y los pesos fetales estimados con los de los grupos de control postnatal y prenatal, respectivamente. El peso al nacer en el percentil 90, o mayor, ocurrió en el $28,2 \%$ del grupo de estudio versus el 9,3\% del grupo de control postnatal $(\mathrm{p}<0,001)$ con un riesgo relativo de 3,0 y un intervalo de confianza del $95 \%$ de 1,9-4,9. Por lo que concluyen que el polihidramnios idiopático se asocia con fetos grandes para la edad gestacional y macrosomía independiente de la diabetes materna. Debido a su etiología común con diabetes gestacional, el polihidramnios a menudo se asocia con macrosomía fetal.

Respecto a los factores perinatales, se encontró que los recién nacidos de sexo masculino tienen más riesgo de nacer con muy alto peso (OR 1,8 IC $95 \%$ 1,6$2,1)$. De la misma manera, varios autores refieren que el sexo masculino es riesgo para tener un macrosómico. Muchos autores han observado la capacidad del sexo masculino para ganar peso rápidamente que las mujeres.

Nkwabong et al. (8) encontraron que los bebés macrosómicos con peso al nacer $\geq 4500 \mathrm{~g}$ se encontraron con mayo incidencia entre el sexo 
masculino que entre el sexo femenino (OR: 1,3, IC del $95 \%$ : 0,6-2,8). Stotland et al. (6) refiere que el sexo masculino es factor de riesgo de tener un macrosómico $(\geq 4000 \mathrm{~g} \mathrm{y} \geq 4500 \mathrm{~g})$ con OR 1,7 (IC $1,6-1,7)$ y $1,9(1,7$ $2,0)$ respectivamente. Jiménez et al. (9) presentan en su estudio de los recién nacidos de $4500 \mathrm{~g}$ - $4999 \mathrm{~g}$ que el $70,7 \%$ eran de sexo masculino. Heiskanen et al. (5) encontraron que hubo una preponderancia de los niños varones entre niños macrosómicos $(65,2 \%)$ frente a los lactantes normosómicos. Oster y Toth (10) encontraron que el sexo masculino es más frecuente en recién nacidos de alto y muy alto peso al nacer con OR 1,5 (1,4 $-1,5)$ y $1,6(1,4-1,8)$ respectivamente.

Se ha encontrado que los que nacen postérmino ( $\geq 42$ semanas de gestación) tienen más riesgo de ser macrosómicos OR 8,2 IC 95 \% 3,7-18,1. En su estudio, Nkwabong et al. (8) encontraron que la edad gestacional al momento del parto influyó en la aparición de macrosomía, ya que los partos posteriores al término ( $>42$ semanas de gestación) se asociaron más con $\geq 4$ $500 \mathrm{~g}$ bebés macrosómicos que los controles (OR 2,3, IC $95 \% 0,9-5,6)$. Algunos autores también encontraron que la gestación prolongada era un factor de riesgo para $\geq 4500$ g de macrosomía. Específicamente, una edad gestacional en el parto $>41$ semanas fue un factor de riesgo, mientras que para otros era una edad gestacional $>42$ semanas.

\section{Conclusión}

Se concluye que la incidencia del recién nacido de muy alto peso al nacer en el Hospital Hipólito Unanue de Tacna durante los años 2008-2017 fue alta y los factores asociados a muy alto peso al nacer fueron factores maternos.

\section{Referencias bibliográficas}

1. Molina O, Monteagudo C. Caracterización perinatal del recién nacido macrosómico. Revista Cubana de Obstetricia y Ginecología.2010; 36(3):313-321

2. Ávila R, Herrera M, Salazar C, Camacho R. Factores de riesgo del recién nacido macrosómico. Pediatría de México 2013; 15(1).

3. Rodríguez N, Martínez T, Martínez R, Garriga M, Ortega M. Obesidad en el escolar con antecedente de macrosomía o alto peso al nacer. Rev. Cubana Invest Bioméd [Internet]. 2009 Jun [citado 2018 Ene 10]; 28(2): Disponible en: shorturl.at/jkyDO

4. Ticona M, Huanco D. Macrosomía fetal en el Perú prevalencia, factores de riesgo y resultados perinatales. Revista Ciencia y Desarrollo de UNJBG. 2006; 1 .

5. Heiskanen N, Raatikainen K, Heinonen S. Fetal Macrosomia. A continuing obstetric challenge. Biol Neonate (2006) 90: 98-103.

6. Stotland N.E., Caughey A.B., Breedc E.M., Escobar
G.J. Risk factors and obstetric complications associated with macrosomía. International Journal of Gynecology and Obstetrics 2004; 87: 220- 226

7. Nassar A, Usta I, Khalil A, Melhem Z, Nakad T, Abu Musa A. Fetal Macrosomia (Z4500 g): Perinatal Outcome of 231 Cases According to the Mode of Delivery. Journal of Perinatology 2003; 23:136-141

8. Nkwabong E, Nzalli G, Fomulu J. What are the Risk Factors for $\geq 4500 \mathrm{~g}$ Macrosomia? Journal of Woman's Reproductive Health - 1(1):1-6.

9. Jiménez S, Pentón R, Cairo V, Cabrera R, Chávez L, Álvarez M. Factores de riesgo maternos y fetales en recién nacidos con macrosomía. Medicentro Electrónica, Cuba 2015; 19.

10. Oster R, Toth E. Longitudinal Rates and Risk Factors for Adverse Birth Weight Among First Nations Pregnancies in Alberta. JANUARY JOGC JANVIER 2016

11. Sohaey R, Nyberg DA, Sickler GK, Williams MA. Idiopathic polyhydramnios: association with fetal macrosomía. Radiology 1994; 190:393-396.

\section{Correspondencia}

luna_2_96@hotmail.com
Fecha de recepción: 10 de octubre de 2019

Fecha de aceptación: 15 de noviembre de 2019 